\title{
Generalised multi-impulsive manoeuvres for optimum spacecraft rendezvous in near-circular orbit
}

\section{Gabriella Gaias*}

GSOC/Space Flight Technology Department, DLR German Aerospace Center,

Münchner Str. 20, 82234 Wessling, Germany

Email: gabriella.gaias@dlr.de

*Corresponding author

\section{Simone D'Amico}

Department of Aeronautics and Astronautics, Stanford University, 496 Lomita Mall, Stanford, CA 94305-4035, USA

Email: damicos@stanford.edu

\section{Jean-Sébastien Ardaens}

GSOC/Space Flight Technology Department, DLR German Aerospace Center, Münchner Str. 20, 82234 Wessling, Germany Email: jean-sebastien.ardaens@dlr.de

\begin{abstract}
This work describes the design of an impulsive manoeuvres' planner meant for onboard autonomous optimum formation flying reconfigurations in near-circular orbit. The whole variation of the relative orbit is stepwise achieved through intermediate configurations, so that passive safety and delta- $\mathrm{v}$ consumption minimisation are pursued. The description of the relative motion is accomplished in terms of relative orbital elements and the reconfiguration plan takes into account mean effects due to the Earth oblateness coefficient and differential drag. Manoeuvres consist of sets of triple tangential impulses and a single out-of-plane burn to establish each intermediate configuration. They are scheduled in time intervals compliant with the user-defined permissible time control windows.
\end{abstract}

Keywords: formation flying; formation reconfiguration; autonomy; relative orbital elements; ROEs.

Reference to this paper should be made as follows: Gaias, G., D'Amico, S. and Ardaens, J-S. (2015) 'Generalised multi-impulsive manoeuvres for optimum spacecraft rendezvous in near-circular orbit', Int. J. Space Science and Engineering, Vol. 3, No. 1, pp.68-88.

Biographical notes: Gabriella Gaias received both her MSc in 2005 and $\mathrm{PhD}$ in 2009 in Aerospace Engineering at the Politecnico di Milano University, Italy. Since 2009, she is working as a Research Engineer in the Space Flight Technology Department at the DLR's German Space Operations Center (GSOC). Her working activities focus on development of AOCS/GNC 


\begin{abstract}
simulation systems for formation-flying and proximity operations applications and software development for vision-based relative navigation. She was involved into the PRISMA mission both as GNC Engineer during mission operations and as experiment developer.
\end{abstract}

Simone D'Amico is an Assistant Professor of Aeronautics and Astronautics at Stanford University, California, USA. He is the Founder and Director of the Stanford's Space Rendezvous Laboratory. He received his PhD in Aerospace Engineering from the Technical University of Delft, The Netherlands and received his BS and MS degrees from Politecnico di Milano, Italy. He has been working as a researcher at the German Aerospace Center (DLR) from 2003 to 2013 in the fields of space flight dynamics, autonomous satellite navigation and control, spacecraft formation-flying, and on-orbit servicing. He gave key contributions to the design, development, and operations of spacecraft formation flying and rendezvous missions such as GRACE, TanDEM-X, and PRISMA for which he received several awards.

Jean-Sébastien Ardaens is a Research Engineer in the field of Autonomous Formation Flight at DLR's German Space Operations Center (GSOC) since 2006. He made relevant contributions to the TanDEM-X and PRISMA missions. His current research activities focus on vision-based formation flying and embedded spaceborne navigation systems.

This paper is a revised and expanded version of a paper entitled 'Generalised multi-impulsive manoeuvres for optimum spacecraft rendezvous' presented at the 5th International Conference on Spacecraft Formation Flying Missions and Technologies (SFFMT), Munich, Germany, 29-31 May 2013.

\title{
1 Introduction
}

Spacecraft formation-flying and on-orbit servicing missions require the capability to establish and reconfigure the relative motion of co-orbiting vehicles in a safe, accurate and fuel-efficient manner. Typical operational scenarios of such distributed space systems prescribe manoeuvring time constraints dictated by the satellite bus and payload needs, by available ground support and ground contact issues. This work addresses the design and development of a flexible manoeuvre planning framework for autonomous optimum formation reconfiguration over a given time interval including user-defined permissible control windows.

Various control methods for spacecraft rendezvous have been presented in literature continuous and discrete, numerical and analytical, for circular and eccentric orbits, using relative Cartesian coordinates and orbital elements. Continuous control strategies are usually employed during the final phases of a rendezvous since a forced motion profile is required, and/or when the spacecraft is equipped with a low-thrust actuation system. Impulsive control is generally preferred at far to mid inter-satellite separation ranges to simplify mission operations and planning and/or to avoid interfering with the payload instruments functioning. Among the proposed discrete strategies, Tillerson et al. (2002), and Tillerson and How (2002) propose fuel-optimal guidance and control strategies based on convex optimisation techniques: a linear programming problem is setup to minimise the weighted sum of the norm-1 of the control inputs. Nevertheless, the time 
discretisation of the fast varying dynamics described in the local Cartesian frame, can lead to prohibitive computational loads for spaceborne microprocessors. In order to allow larger time steps, Larsson et al. (2006) apply this methodology making use of the Yamanaka-Ankersen state transition matrix (Yamanaka and Ankersen, 2002), though neglecting the modelling of $J_{2}$. General limitations of this approach are represented by the limited predictability of the timing of control correction manoeuvres and the difficulty to introduce manoeuvres' exclusion windows.

Analytical impulsive control techniques are often based on the exploitation of the Gauss variational equations (GVE). Examples are provided by Schaub and Alfriend (2001), and Vaddi et al. (2005). Ichimura and Ichikawa (2008) introduce a parametrisation defined from the analytical solution of the Hill-Clohessy-Wiltshire (HCW) equations to develop an analytical open-time minimum fuel reconfiguration strategy. This prescribes three in-plane impulses to achieve optimal reconfigurations in the case that the aimed change in the size of the formation is greater than the change in the drift and enough transfer time is available. Starting from this open-loop profile, they designed a suboptimal feedback controller.

The most recent flight demonstrations of autonomous formation control are represented by the experiments on the PRISMA mission (Bodin et al., 2012), among them the Spaceborne Autonomous Formation Flying Experiment (SAFE) (D'Amico et al., 2012) and by the TanDEM-X Autonomous Formation Flying (TAFF) system (Ardaens et al., 2011). These systems rely on practical and simple closed-form solutions of the GVE equations which can be expressed analytically and be efficiently embedded into spaceborne microprocessors.

TAFF makes use of pairs of (anti-)along-track manoeuvres separated by half an orbital revolution for in-plane formation keeping only, whereas SAFE is able to exploit radial and cross-track pulses for enhanced in-plane and out-of-plane control respectively. The logic which triggers the execution of manoeuvres is based either on a fixed manoeuvre cycle (TAFF) or on the violation of predefined control windows defined about nominal relative orbital elements (ROEs) (SAFE). Although the necessary manoeuvres are autonomously planned and executed onboard based on the most recent estimates of the ROEs, the nominal or desired formation configurations (i.e., guidance) are still prescribed from ground through telecommands and thus with man-in-the-loop.

This work builds on the state-of-the-art in an attempt to generalise the SAFE and TAFF algorithms in order to improve the autonomy (including guidance) and flexibility (including constraints) of flight-proven methodologies without sacrificing simplicity and determinism to the largest possible extent. The proposed methodology focuses on formations with an active chief satellite in near-circular orbit about an oblate Earth and perturbed by aerodynamic drag. Nevertheless, the approach is kept as general as possible to allow for future improvements in the perturbation modelling and/or extension to eccentric orbits.

In contrast to previous flight systems which rely on GPS relative navigation, here observability issues related to angles-only navigation are taken into account to support far-range non-cooperative rendezvous scenarios as well. The main goal is to compute the guidance profile onboard as a sequence of intermediate desired formation configurations pursuing certain optimality criteria. This translates into an optimisation problem where an aimed final formation has to be acquired in a defined time period while maximising the satisfaction of the prescribed criteria. Operationally, the minimisation of the total delta- $\mathrm{v}$ consumption and the maximisation of the navigation system observability are sought. 
This work presents the core algorithm embedded in the autonomous manoeuvre planner module for the autonomous vision approach navigation and target identification (AVANTI) experiment (Gaias et al., 2014a), schedule for execution within the DLR FireBird mission (Reile et al., 2013). AVANTI is intended to demonstrate vision-based non-cooperative autonomous approach and recede manoeuvring making use of angles-only measurements. Therefore, the active servicer satellite performs some proximity operations with respect to a picosatellite, previously released in-orbit through a deployment device.

This paper is organised as follows. First of all, the overall concept of the planner is introduced. The central part of the paper deals with the mathematical description of the employed algorithms for the guidance and control problems. The objective of the guidance, addressed in Section 3, is to provide a set of intermediate relative orbits to be acquired at certain intermediate times (related to the schedule of the allowed manoeuvre windows) in order to achieve the final aimed relative state. The local control task discussed in Section 4, instead, deals with the problem of establishing any intermediate relative geometry in a limited and fixed time period. Before concluding, Section 6 provides an overview of the whole planner implementation, whereas an example showing the functioning of the planner is provided and discussed in Section 7.

\section{Overall concept}

The objective of the generalised multi-impulsive manoeuvres controller is to provide the sequence of manoeuvres needed starting from an initial time to achieve a prescribed relative orbit at a given final time. Thus, the controller prototyped in this research delivers an open-loop manoeuvre profile that covers the whole reconfiguration's horizon.

In agreement and continuation of previous work conducted by the authors, ROEs are used to parametrise the relative dynamics. They consist of a set of six elements with an immediate geometrical meaning of the characteristics of the relative orbit that they represent. They are defined as:

$$
\delta \alpha=\left(\begin{array}{c}
\delta a \\
\delta \lambda \\
\delta e_{x} \\
\delta e_{y} \\
\delta i_{x} \\
\delta i_{y}
\end{array}\right)=\left(\begin{array}{c}
\delta a \\
\delta \lambda \\
\delta e \cos \varphi \\
\delta e \sin \varphi \\
\delta i \cos \theta \\
\delta i \sin \theta
\end{array}\right)=\left(\begin{array}{c}
\left(a-a_{\mathrm{d}}\right) / a_{\mathrm{d}} \\
u-u_{d}+\left(\Omega-\Omega_{\mathrm{d}}\right) \cos i_{\mathrm{d}} \\
e \cos \omega-e_{\mathrm{d}} \cos \omega_{\mathrm{d}} \\
e \sin \omega-e_{\mathrm{d}} \sin \omega_{\mathrm{d}} \\
i-i_{\mathrm{d}} \\
\left(\Omega-\Omega_{\mathrm{d}}\right) \sin i_{\mathrm{d}}
\end{array}\right)
$$

where $a, e, i, \omega, \Omega$, and $M$ denote the classical Keplerian elements, and $u=M+\omega$ is the mean argument of latitude. The subscript ' $d$ ' labels the deputy spacecraft of the formation, which, in this work, plays the role of the manoeuvrable servicer satellite. In the sequel all absolute quantities refer to the servicer satellite, thus the subscript is dropped. The quantities $\delta \mathbf{e}=(\delta e \cos \varphi \delta e \sin \varphi)^{\mathrm{T}}$ and $\delta \mathbf{i}=(\delta i \cos \theta \delta i \sin \theta)^{\mathrm{T}}$ define the relative eccentricity and inclination vectors. Under the assumptions of the HCW equations (Clohessy and Wiltshire, 1960), their magnitudes $\delta e$ and $\delta i$ express the in-plane and out-of-plane amplitudes of the oscillations of the relative motion respectively. The phase angles $\varphi$ and $\theta$ identify the relative perigee and relative ascending node of the relative 
orbit. Therefore, $\delta \mathbf{e}$ and $\delta \mathbf{i}$ fully describe the orientation and the shape of the relative motion. The remaining ROEs, the relative semi-major axis $\delta a$ and the relative mean longitude $\delta \lambda$ provide the mean offsets in local radial and local along-track directions of the orbital frame respectively. When both the satellites belong to Keplerian orbits, only the element $\delta \lambda$ varies with time according to:

$$
\delta \lambda(t)=-1.5 n\left(t-t_{0}\right) \delta a_{0}+\delta \lambda_{0}
$$

thus $\delta a$ embodies the drift coefficient of the relative motion, $n$ is the servicer mean angular motion, and ' 0 ' labels the quantities at the initial time. The detailed description of the ROEs, together with the development and discussion of the model of the relative dynamics and its extension to include differential drag and secular effects due to the $J_{2}$ perturbation is given in D'Amico (2010). This topic is further addressed in Section 3.

By letting $\mathbf{P}$ identifying the dimensional point in the ROE space (i.e., $\mathbf{P}(t)=a \delta \alpha(t)$ ), a reconfiguration from a certain initial relative orbit to an aimed final one can be defined as the transition $\mathbf{P}_{0} \rightarrow \mathbf{P}_{\mathrm{F}}$ over the finite time interval $\left[t_{0}, t_{\mathrm{F}}\right]$. The main concept of the controller consists in splitting the reconfiguration problem into two layers:

1 guidance, that is the computation of some (optimal with respect to a given criterion) intermediate configurations $\mathbf{P}_{i}$ to be reached at the given times $t_{i}$, in order to achieve the final aimed $\mathbf{P}_{\mathrm{F}}$.

2 control, that is the local resolution of the $i^{\text {th }}$ problem of establishing any intermediate $\mathbf{P}_{i}$ in a limited (and specified) time horizon that ends at $t_{i}$.

The idea that motivates such a layered approach is to simplify the resolution of the problem by decoupling the geometry of the relative orbits from the computation of the manoeuvres to establish such motions. Thus, as seen by the guidance layer, the ROEs evolve according to the model of the dynamics until some discontinuities occur. These represent the global effect of the bunch of manoeuvres that would be needed to accomplish such jumps of ROEs. Therefore, if each manoeuvres set allows exactly achieving the corresponding intermediate state $\mathbf{P}_{i}$, then the guidance-control decoupling introduces no error on the final orbit $\mathbf{P}_{\mathrm{F}}$. At the same time, at the control level, the manoeuvres have to be computed pursuing the same optimality criterion employed by the guidance layer, in order to avoid worsening the quality of the solution. It is emphasised that, this approach allows immediately recognising un-safe or un-feasible configurations, since they correspond to $\mathbf{P}_{i}$ points in forbidden regions of the state space. Whenever the merit criterion of the planning problem can be written as function of the variations of ROEs, then the guidance output comes from the solution of an optimum problem where the variations of ROEs that have to occur over each allowed manoeuvre window are taken as control variables.

On the other hand, the local problem focuses on the computation of the locations and the magnitudes of the manoeuvres that allow achieving an aimed variation of ROEs. At this step of the process, the dynamics can be assumed as Keplerian, thus the out-of-plane and in-plane motions are decoupled. The out-of-plane reconfiguration reduces to the deterministic problem of computing the cross-track thrust and its burn time to realise a given change in the relative inclination vector. The in-plane reconfiguration problem, instead, requires at least two burns in order to realise any given change in the four in-plane ROEs. This translates into an underdetermined system with six unknowns (i.e., time, radial and tangential delta-v magnitudes for each manoeuvre) in four equations. 
Gaias and D'Amico (2014) show that two impulses reconfiguration schemes require a numerical solution method in order to realise any given set of in-plane ROEs variation. Fully analytical solution schemes exist when three impulses are used instead. Specifically these employ pure tangential manoeuvres that occur at half orbital period multiples of the mean argument of latitude equal to the phase angle of the total aimed variation of the relative eccentricity vector. This topic is further tackled in Section 4.

Figure 1 provides a schematic description of the layered structure of the planner: the main tasks of each layer are specified. Concerning the interfaces to the controller, inputs consist of the absolute state of the servicer $\mathbf{y}_{0}$ (i.e., position and velocity in the inertial J2000 reference frame), the so far estimated set of ROEs $\mathbf{P}_{0}$ (both at the initial time $t_{0}$ ), the aimed final orbit $\mathbf{P}_{\mathrm{F}}$, and the final time $t_{\mathrm{F}}$. Time constraints of an operative scenario are represented by: time intervals in which it is not allowed manoeuvring, the minimum time from the generation of the manoeuvres profile to the first command to the thrusters, and the minimum time spacing between different manoeuvres to let the spacecraft being prepared with proper attitude and thrusters' state. Other typical operational constraints are the minimum delta- $v$ impulse, the maximum allowed magnitude of a delta- $v$, the minimum inter-satellite separations respectively perpendicular and aligned to the flight direction (related to passive safety), and, in case of vision-based rendezvous, target visibility constraints (D'Amico et al., 2013). Depending on the desired operative mode (see Section 2.1), the manoeuvre planner foresees a reconfiguration in only one step (minimum delta- $v$ mode) or plans intermediate configurations delimited by the forbidden time intervals defined by the user (maximum observability mode).

Figure 1 Structure of the manoeuvre planner (see online version for colours)
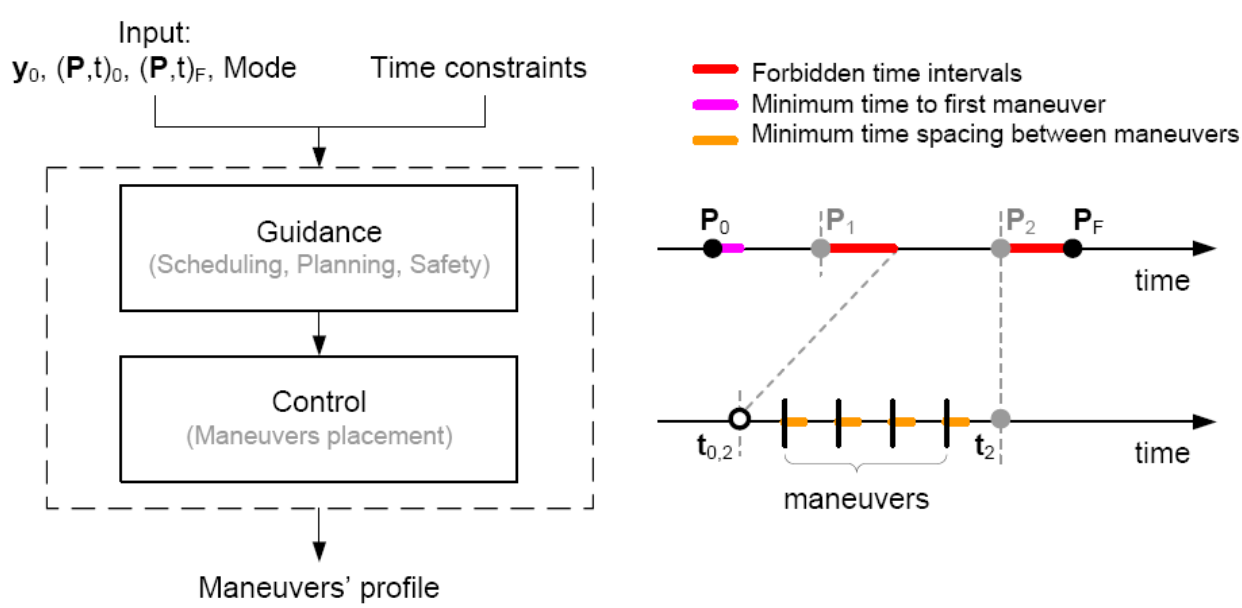

\subsection{Operative modes}

The lower bound of the delta-v expenditure to accomplish an in-plane formation reconfiguration is known (Eckstein, 1992; Ichimura and Ichikawa, 2008; Gaias and D'Amico, 2014): it requires to exploit only tangential burns. By using three impulses, an analytical solution exists for any set of aimed correction of in-plane ROEs. As explained in Gaias and D'Amico (2014), in the typical scenario of a rendezvous, this 
triple-tangential manoeuvre solution is able to achieve the absolute minimum of the delta-v cost. With rendezvous scenario, in fact, it is meant to shrink the relative motion while approaching the target, aiming to a bounded final motion. This is the case in which the change in the shape of the formation $(\|\Delta \delta \mathbf{e}\|)$ is dominant with respect to the change in the generalised drift $\left(\Delta \delta a^{*}\right)$ since large relative mean longitude transfers occur over long time horizons. The actual computation of the manoeuvres is presented later in Section 4. Regarding the guidance layer, instead, if the planning criterion is to seek for the minimum delta- $\mathrm{v}$, then no intermediate $\mathbf{P}_{i}$ should be introduced and manoeuvres should be placed in agreement with the scheme of Section 4 . This objective is referred as minimum delta- $v$ operative mode.

Although this mode guarantees to spend the minimum delta-v consumption, from an operative point of view this approach presents some not practical aspects. First of all, when a servicer satellite approaches a non-cooperative target the errors in the estimated initial relative state $\mathbf{P}_{0}$ are large (D'Amico et al., 2013). Thus, it is typically necessary to update the manoeuvres' profile as soon as the knowledge of the relative state is refined. Consequently, it is not convenient to perform large (due to the limited number of impulses) manoeuvres at the beginning of the rendezvous. Secondly, taking into account the effects of the manoeuvre execution errors, it is not efficient to accomplish a reconfiguration thorough few large manoeuvres. On the other hand, if the relative navigation is performed via angles-only measurements the most fruitful way to improve the observability properties of the system is to perform some manoeuvres; the more intense the manoeuvre activity the more observable the relative navigation problem (Gaias et al., 2014b). To this end, it is not convenient to spread the few available impulses over wide time horizons. In an attempt to overcome these practical issues while still pursuing to minimise the fuel consumption during the rendezvous, a maximum observability operative mode is designed.

According to it, the rendezvous is accomplished in a step-wise manner through a user-defined number of intermediate configurations to be reached at certain times $t_{i}$ that are the end time of the windows in which it is allowed manoeuvring (see $\left[t_{0, i}, t_{i}\right]$ in Figure 1). The ROE sets at such intermediate times are computed so that the total delta-v is minimised (see Section 3). Clearly, having introduced intermediate $\mathbf{P}_{i}$ the maximum observability mode consumes more delta- $\mathrm{v}$ than the minimum delta- $\mathrm{v}$ one. Nevertheless, the user can now intensify the occurrence of manoeuvres when convenient due to practical motivations. Therefore, the input that lists the forbidden time intervals can also be used to allow a sort of control action of the user over the manoeuvres' profile. Moreover, the introduction of intermediate configurations has the positive benefit to strengthen the supervision over safety during the approach. To this aim, it is simply needed to verify, and eventually to correct, that the $\mathbf{P}_{i}$ output by the planner have an adequate relative eccentricity and inclination vectors separation (D'Amico and Montenbruck, 2006).

According to the structure of the manoeuvre planner depicted in Figure 1, both the operative modes make use of the same local control functionality. In other words, if the forbidden time intervals input is left empty, the observability mode coincides with the minimum delta-v one. Nevertheless, at implementation level, within observability mode no a-posteriori check on the feasibility of the manoeuvres' location has to be performed, 
as all the reconfiguration time horizon is free from forbidden regions (see Section 6.3). A further synergy between the two operative modes is provided by the typology of manoeuvres employed. In Gaias et al. (2014b), in fact, it was shown that once fixed the magnitude of a manoeuvre, tangential burns are to be preferred with respect to the radial ones in terms of effects on the observability of the relative navigation problem. This is due to the fact that the drift translates into a more distinguishable pattern of one of the two measured angles with respect to the trend before the manoeuvre.

\section{The planning problem}

This section deals with the solution of the problem of finding $m$ intermediate relative orbits $\mathbf{P}_{i}$ to be reached at the times $t_{i}$ in order to perform the reconfiguration $\mathbf{P}_{0} \rightarrow \mathbf{P}_{\mathrm{F}}$ while using the minimum possible delta-v. According to the employed solution method, all the intermediate times will be exploited.

The delta-v cost of the plan can be expressed by the following convex form:

$$
J_{\text {plan }}=\sum_{i=1}^{m}(\Delta\|\delta \mathbf{i}\|)_{i}^{2}+\sum_{i=1}^{m}(\Delta \delta a)_{i}^{2}+\sum_{i=1}^{m}(\Delta \delta \lambda)_{i}^{2}+\sum_{i=1}^{m}(\Delta\|\delta \mathbf{e}\|)_{i}^{2}
$$

where the $i^{\text {th }}$ variations of ROEs $(\Delta \delta \alpha)_{i}$ that occur at $t_{i}$ are assumed as control variables. Within the hypothesis of linearised relative motion, the closed form solution of the mean relative motion in the presence of $J_{2}$ Earth oblateness effects is presented in D'Amico (2010, p.34). The interaction between the satellites and the upper layers of the atmosphere is modelled through a differential drag, which produces an off-set in the along-track direction proportional to the square of the elapsed time, when assuming the atmospheric density constant within few kilometres. Taking into account the property of the relative dynamics expressed by equation (2), the effect of the differential drag is introduced in the linear model of the relative dynamics as a linear variation of the relative semi-major axis with respect to the elapsed time D'Amico (2010, p.37):

$$
\delta \dot{a}=-\frac{1}{n} \Delta B \rho v^{2}
$$

where $\Delta B$ is the difference of the ballistic coefficients of the satellites and $\rho$ and $v$ are respectively the atmospheric density and the deputy velocity with respect to the atmosphere. The correspondent complete state transition matrix can be written augmenting the ROE state of equation (1) with $\delta \dot{a}$ :

$$
\left(\begin{array}{l}
\delta \dot{a} \\
\delta \boldsymbol{\alpha}
\end{array}\right)_{\mathrm{F}}=\boldsymbol{\Phi}_{F, 0}\left(\begin{array}{l}
\delta \dot{a} \\
\delta \boldsymbol{\alpha}
\end{array}\right)_{0}
$$

where the subscripts indicate the transition from the initial to the final time, according to: 


$$
\begin{aligned}
& \Phi_{F, 0}=\left[\begin{array}{ccccccc}
1 & 0 & 0 & 0 & 0 & 0 & 0 \\
\left(t_{F}-t_{0}\right) & 1 & 0 & 0 & 0 & 0 & 0 \\
\frac{v}{2}\left(t_{F}-t_{0}\right)^{2} & v\left(t_{F}-t_{0}\right) & 1 & 0 & 0 & 0 & 0 \\
0 & 0 & 0 & 1 & -\dot{\varphi}\left(t_{F}-t_{0}\right) & 0 & 0 \\
0 & 0 & 0 & \dot{\varphi}\left(t_{F}-t_{0}\right) & 1 & 0 & 0 \\
0 & 0 & 0 & 0 & 0 & 1 & 0 \\
0 & 0 & 0 & 0 & 0 & \lambda\left(t_{F}-t_{0}\right) & 1
\end{array}\right] \\
& v=-\frac{3}{2} n \quad \dot{\varphi}=\frac{3}{n} n \gamma\left(5 \cos ^{2} i-1\right) \quad \mu=-\frac{21}{2} n \gamma \sin 2 i \quad \lambda=3 n \gamma \sin ^{2} i
\end{aligned}
$$

where $\gamma$ is the following function of the $J_{2}$ coefficient, the orbit altitude, the Earth radius $R_{\oplus}$, and an eccentricity factor $\eta=\sqrt{1-e^{2}}$ :

$$
\gamma=\frac{J_{2}}{2} \frac{R_{\oplus}^{2}}{a^{2} \eta^{4}}
$$

At the guidance level the evolution $\mathbf{P}_{0} \rightarrow \mathbf{P}_{\mathrm{F}}$ is given by:

$$
\mathbf{P}_{\mathrm{F}}=\boldsymbol{\Phi}_{F, 0} \mathbf{P}_{0}+\Phi_{F, 1} a(\Delta \delta \boldsymbol{\alpha})_{1}+\cdots+\Phi_{F, m} a(\Delta \delta \boldsymbol{\alpha})_{m}
$$

Taking into account the structures of equation (3) and equation (6), the optimum problem of minimising $J_{\text {plan }}$ while satisfying the end conditions equation (8), consists in two disjointed subproblems in the subsets $\left(\Delta \delta \dot{a}, \Delta \delta a, \Delta \delta \lambda, \Delta \delta i_{x}, \Delta \delta i_{y}\right)$ and $\left(\Delta \delta e_{x}, \Delta \delta e_{y}\right)$.

They can be solved in the same way by rearranging equation (8) as follows:

$$
\begin{aligned}
& {[\underbrace{\boldsymbol{\Phi}_{\mathrm{F}, 1}^{\delta^{*}} \cdots \boldsymbol{\Phi}_{\mathrm{F}, m-1}^{\delta^{*}} \boldsymbol{\Phi}_{\mathrm{F}, m}^{\delta^{*}} \cdots \underbrace{\boldsymbol{\Phi}_{\mathrm{F}, 1}^{\delta^{*}} \cdots \boldsymbol{\Phi}_{\mathrm{F}, m-1}^{\delta^{*}} \boldsymbol{\Phi}_{\mathrm{F}, m}^{\delta^{*}}}_{\text {last column }}}_{\text {first column }}]\left(\begin{array}{c}
x_{1,1} \\
\vdots \\
x_{1, m} \\
\vdots \\
x_{p, 1} \\
\vdots \\
x_{p, m}
\end{array}\right)=\mathbf{b}_{0}} \\
& \mathbf{b}_{0}=\mathbf{P}_{\mathrm{F}}-\Phi_{\mathrm{F}, 0} \mathbf{P}_{0}
\end{aligned}
$$

where the vectors $\left(\mathbf{x}_{1}, \cdots, \mathbf{x}_{p}\right)$ on turn assume the meaning of $\left(a \Delta \delta \dot{a}, a \Delta \delta a, a \Delta \delta \lambda, a \Delta \delta i_{x}\right.$, $a \mathbf{\Delta} \delta i_{y}$ ) and ( $\left.a \mathbf{\Delta} \delta e_{x}, a \mathbf{\Delta} \delta e_{y}\right)$. As long as equation (4) is constant over a reconfiguration, the equations in $\Delta \delta \dot{a}$ do not bring any information. Thus, the effect of the differential drag is condensed in $\mathbf{b}_{0}$, that is in the total change of the $\delta a$ and $\delta \lambda$ components, in agreement with D'Amico (2010).

The remaining equations constitute the two subproblems with $p=4$ and $p=2$ respectively. As a first step, equation (8) is used to express the $m^{\text {th }}$ ROE variations (i.e., $\left.x_{j, m}\right)$ as function of the first $m-1$ referred as $\tilde{\mathbf{x}}_{j}$. By substituting them back in equation 
(3), also the merit function depends only on these $m-1$ jumps. The necessary conditions for optimality are:

$$
\frac{\partial J_{\text {plan }}}{\partial \tilde{\mathbf{x}}_{j}}=0_{1 \times m-1}^{\mathrm{T}} \quad j=1, \cdots, p
$$

Due to the forms of the merit function and of the variables' domain, these are also the sufficient conditions to identify the unique minimum of $J_{\text {plan }}$. Moreover, the optimal solution reduces to solve a linear system in the problem's variables. This means that the particular choice of the parametrisation allows solving the optimum problem for the dynamical system as a geometrical problem, whose solution is the most convenient succession of reachable points $\mathbf{P}_{i}$ in the ROE space.

The transition matrix of the $\delta a / \delta \lambda / \delta \mathbf{i}$ subproblem satisfies the following property:

$$
\Phi\left(t_{j}, t_{i}\right) \cdot \Phi\left(t_{i}, t_{k}\right)=\Phi\left(t_{j}, t_{k}\right)
$$

thus, the solution of equation (10) is given by:

$$
\begin{aligned}
& \left(\begin{array}{c}
\tilde{\mathbf{x}}_{1} \\
\vdots \\
\tilde{\mathbf{x}}_{4}
\end{array}\right)_{o p t}=\left[\begin{array}{cccc}
\mathbf{I}+\eta \mathbf{A}+v^{2} \mathbf{C} & v \mathbf{D} & v \mu \mathbf{C} & \mathbf{O} \\
v \mathbf{D}^{\mathrm{T}} & \mathbf{I}+\mathbf{A} & \mu \mathbf{D}^{\mathrm{T}} & \mathbf{O} \\
\mu \nu \mathbf{C} & \mu \mathbf{D} & \mathbf{I}+\eta \mathbf{A}+\mu^{2} \mathbf{C}+\lambda^{2} \mathbf{C} & \lambda \mathbf{D} \\
\mathbf{O} & \mathbf{O} & \lambda \mathbf{D}^{\mathrm{T}} & \mathbf{I}+\mathbf{A}
\end{array}\right]^{-1} \mathbf{b}_{0} \\
& \mathbf{b}_{0}=\left(\begin{array}{c}
b_{0,1} \tilde{\mathbf{a}}+v b_{0,2}^{*} \tilde{\mathbf{c}} \\
b_{0,2}^{*} \tilde{\mathbf{a}} \\
b_{0,3} \tilde{\mathbf{a}}+\left(\mu b_{0,2}^{*}+\lambda b_{0,4}^{*}\right) \tilde{\mathbf{c}} \\
b_{0,4}^{*} \tilde{\mathbf{a}}
\end{array}\right) \\
& b_{0,2}^{*}=b_{0,2}-v b_{m} b_{0,1}-\mu b_{m} b_{0,3} \\
& b_{0,4}^{*}=b_{0,4}-\lambda b_{m} b_{0,3}
\end{aligned}
$$

The matrix quantities are $\mathbf{A}=\tilde{\mathbf{a}} \tilde{\mathbf{a}}^{\mathrm{T}}, \mathbf{C}=\tilde{\mathbf{c}} \tilde{\mathbf{c}}^{\mathrm{T}}, \mathbf{D}=\tilde{\mathbf{c}} \tilde{\mathbf{a}}^{\mathrm{T}}$, and the so far unmentioned notation is defined as:

$$
\tilde{\mathbf{a}}_{m-1 \times 1}=\left(\begin{array}{c}
1 \\
\vdots \\
1
\end{array}\right) \quad \mathbf{b}_{m \times 1}=\left(\begin{array}{c}
t_{F}-t_{1} \\
\vdots \\
t_{F}-t_{m}
\end{array}\right)=\left(\begin{array}{c}
\tilde{\mathbf{b}}_{m-1 \times 1} \\
b_{m}
\end{array}\right) \quad \tilde{\mathbf{c}}=\tilde{\mathbf{b}}-b_{m} \tilde{\mathbf{a}}
$$

where $\tilde{\bullet}$ labels vectors of dimension $m-1$.

The time composition of state transition matrix of the relative eccentricity vector subproblem is instead:

$$
\begin{aligned}
\Phi^{\delta \mathrm{e}}\left(t_{j}, t_{i}\right) \cdot \boldsymbol{\Phi}^{\delta \mathrm{e}}\left(t_{i}, t_{k}\right) & =\Phi^{\delta \mathrm{e}}\left(t_{j}, t_{k}\right)-\dot{\varphi}^{2}\left(t_{j}-t_{i}\right)\left(t_{i}-t_{k}\right) \cdot \mathbf{I}_{2 \times 2} \\
& \doteq \tilde{\Phi}^{\delta \mathrm{e}}\left(t_{j}, t_{k}\right)
\end{aligned}
$$

In order to lead back $\delta \mathbf{e}_{0} \rightarrow \delta \mathbf{e}_{\mathrm{F}}$ to the form of equation (9), the following approximation is introduced: 


$$
\boldsymbol{\Phi}^{\delta \mathbf{e}}\left(t_{h}, t_{j}\right) \cdot \tilde{\boldsymbol{\Phi}}^{\delta \mathbf{e}}\left(t_{j}, t_{k}\right) \approx \tilde{\boldsymbol{\Phi}}^{\delta \mathbf{e}}\left(t_{h}, t_{k}\right)
$$

coherently all the matrices that compare in equation (9), with exception of $\Phi_{\mathrm{F}, m}^{\delta \mathrm{e}}$, become of the type defined in equation (14). This approximation starts introducing some errors from the third manoeuvre window on. Terms of $\dot{\varphi}^{2} \Delta t^{2}$ are neglected with respect to the unit diagonal, and terms $\dot{\varphi}^{3} \Delta t^{3}$ are neglected in the extra-diagonal components, since $\dot{\varphi} \Delta t \approx 10^{-3}$ for a Sun-synchronous, $500 \mathrm{~km}$ high orbit and $\Delta t$ of few orbital periods.

By keeping $\tilde{\mathbf{a}}$, modifying $\mathbf{b}=\dot{\varphi} \mathbf{b}$, and introducing these further vectors:

$$
\begin{gathered}
\tilde{\mathbf{d}}_{m-1 \times 1}=\dot{\varphi}\left(\begin{array}{c}
t_{m}-t_{1} \\
\vdots \\
t_{m}-t_{m-1}
\end{array}\right) \quad \tilde{\mathbf{d}}_{m-1 \times 1}=\dot{\varphi}\left(\begin{array}{c}
t_{m-1}-t_{1} \\
\vdots \\
t_{m-1}-t_{m-1}
\end{array}\right) \\
\tilde{\mathbf{r}}=\tilde{\mathbf{a}}-b_{m} \tilde{\mathbf{d}} \text { if } t_{\mathrm{F}}>t_{m} \quad \tilde{\mathbf{r}}=\tilde{\mathbf{a}}-\dot{\varphi}\left(t_{m}-t_{m-1}\right) \tilde{\mathbf{d}} \text { if } t_{\mathrm{F}}=t_{m}
\end{gathered}
$$

the solution of the optimum problem becomes:

$$
\left(\begin{array}{l}
\tilde{\mathbf{x}} \\
\tilde{\mathbf{y}}
\end{array}\right)_{o p t}=\left[\begin{array}{cc}
\mathbf{I}+\mathbf{F}+\mathbf{G} & \mathbf{H}-\mathbf{H}^{\mathrm{T}} \\
\mathbf{H}^{\mathrm{T}}-\mathbf{H} & \mathbf{I}+\mathbf{F}+\mathbf{G}
\end{array}\right]^{-1}\left(\begin{array}{c}
-\frac{b_{x 0}+b_{m} b_{y 0}}{1+b_{m}^{2}} \tilde{\mathbf{f}}+\frac{b_{y 0}-b_{m} b_{x 0}}{1+b_{m}^{2}} \tilde{\mathbf{g}} \\
-\frac{b_{x 0}+b_{m} b_{y 0}}{1+b_{m}^{2}} \tilde{\mathbf{g}}-\frac{b_{y 0}-b_{m} b_{x 0}}{1+b_{m}^{2}} \tilde{\mathbf{f}}
\end{array}\right)
$$

where $\mathbf{F}=\tilde{\mathbf{f}} \tilde{\mathbf{f}}^{\mathrm{T}}, \mathbf{G}=\tilde{\mathbf{g}} \tilde{\mathbf{g}}^{\mathrm{T}}$, and $\mathbf{H}=\tilde{\mathbf{f}} \tilde{\mathbf{g}}^{\mathrm{T}}$ with:

$$
\tilde{\mathbf{f}}=-\frac{1}{1+b_{m}^{2}}\left(\tilde{\mathbf{r}}+b_{m} \tilde{\mathbf{b}}\right) \quad \tilde{\mathbf{g}}=\frac{1}{1+b_{m}^{2}}\left(\tilde{\mathbf{b}}-b_{m} \tilde{\mathbf{r}}\right)
$$

To conclude this section, the intermediate relative configurations output by the planning problem are computed from equation (8), once obtained the optimal variations of ROEs from equations (12) and (17).

It is emphasised that, due to the structure of the state transition matrix, one could solve this planning problem via a geometrical method which stepwise covers the aimed total variation of ROEs through corrections proportional to the covered time portion. The result obtained would be the same as long as $J_{\text {plan }}$ describes the delta-v in terms of the size of the ROE total jump. Nevertheless, the proposed method offers a rigorous approach that shows that such solution guarantees the minimum delta-v, being as well suitable for an automated implementation. Moreover, this approach provides a working frame ready to support possible improvements, as for example, introducing constraints directly at the planning level, as penalties function of the variations of $\operatorname{ROE}(\Delta \delta \alpha)_{i}$.

Finally, thanks to the exploitation of the state transition matrix of equation (6), this approach generalises the results of Eckstein (1992), and Ichimura and Ichikawa (2008), including the effects of $J_{2}$ and differential drag perturbations. 


\section{The local control problem}

This section addresses the problem to compute magnitude and location of the manoeuvres required to achieve a certain $\mathrm{ROE}$ variation over a finite time frame.

The local control problem solves a fixed-time, fixed-end-condition problem. Type and number of impulses are respectively fixed by minimum delta-v considerations and by the existence of an analytical solution, accordingly to the design directions provided in Gaias and D'Amico (2014).

At each local problem, the manoeuvres shall accomplish the following variation of ROEs over the time span $\left[t_{0 i}, t_{i}\right]$ :

$$
a \Delta \delta \tilde{\boldsymbol{\alpha}}=a \delta \boldsymbol{\alpha}_{i}-\boldsymbol{\Phi}_{i, 0 i} a \delta \boldsymbol{\alpha}_{0, i}
$$

where the variation takes into account the whole effect of the orbit perturbations throughout the $\left[t_{0 i}, t_{i}\right]$ interval of time.

To compute the manoeuvres, instead, the hypothesis of Keplerian motion is fully acceptable, thus neglecting the differential perturbations effect related to the changes in the relative orbits operated by the manoeuvres. These in fact determine negligible variations in the mean arguments of latitude of the manoeuvres.

Within the Keplerian motion, the out-of-plane correction is decoupled from the inplane one. It is obtained by a single manoeuvre placed either at $u_{\text {oop }}$ or at $u_{\text {oop }}+\pi$ according to:

$$
u_{\text {oop }}=\arctan \left(\frac{\Delta \delta \tilde{i}_{y}}{\Delta \delta \tilde{i}_{x}}\right) \text { with }\left|\delta v_{n}\right|=n a\|\Delta \delta \tilde{\mathbf{i}}\|
$$

where the sign of $\delta v_{n}$ is chosen so that the last two end-conditions of equation (19) are satisfied.

The relations between an instantaneous velocity increment in the Hill's orbital frame and the consequent change of ROEs are obtained from the inversion of the adopted linear model. As explained in Gaias and D'Amico (2014), a minimum of two manoeuvres are required to meet a whatever in-plane end-conditions' set. Thus, by assuming as problem variables the magnitudes and locations of such manoeuvres, the local control in-plane subproblem to satisfy equation (19) is underdetermined. As long as we are interested in solutions that minimise the delta-v consumption, the size of the solution space can be reduced by employing only tangential burns, since they allow consuming less delta-v while affecting all the in-plane ROE terms, either instantaneously or within a certain timespan. On the one hand, if only two tangential burns were used, it would not be always possible to satisfy all the possible end-conditions' sets. On the other hand, by employing three tangential impulses an analytical solution of equation (19) always exists, upon the condition of performing such manoeuvres at integer half orbital period multiples of a fixed mean argument of latitude. In particular the satisfaction of equation (19) implies that such required location is exactly the phase angle of the aimed variation of the relative eccentricity vector:

$$
u=\arctan \left(\frac{\Delta \delta \tilde{e}_{y}}{\Delta \delta \tilde{e}_{x}}\right) \quad u_{\mathrm{ipj}}=\bar{u}+k_{j} \pi, \quad j=1 \ldots 3 \quad k_{1}<k_{2}<k_{3}
$$


In the limited time horizon of each control window, if lasting more than one and a half orbital periods, there exist a finite number of possible solutions, depending on the values assumed by $k_{j}$. The correspondent delta-v quantities are computed according to Tables 1 and 2. There the following notation is adopted:

$$
\begin{aligned}
& a \Delta \delta \tilde{\boldsymbol{\alpha}}_{\mathrm{ip}}=(A, L, E, F)^{\mathrm{T}} \\
& q=u_{i}-u_{\mathrm{ip} 1} \quad p=u_{i}-u_{\mathrm{ip} 2} \quad l=u_{i}-u_{\mathrm{ip} 3}
\end{aligned}
$$

where $u_{i}$ represents the end-time limit $t_{i}$ of the control window. The implementation logic to select one solution among all these possible ones is explained in Section 6.3.

\begin{tabular}{|c|c|c|c|c|c|}
\hline \multicolumn{3}{|c|}{ Aimed end-conditions } & \multirow{2}{*}{$\begin{array}{c}\text { Choice of } k_{j} \\
\text { Signs }\end{array}$} & \multicolumn{2}{|c|}{$\delta \tilde{v}=\frac{b A+g B}{4 b}$} \\
\hline & $B$ & $b$ & &,,+----+-+- &,,+-+++--++ \\
\hline $\bar{u} \in[0, \pi / 2)$ & $\mathrm{E}$ & $\cos \bar{u}$ & $\operatorname{sign}\left(\cos u_{\mathrm{ip} j}\right)$ & $g=1$ & $g=-1$ \\
\hline $\bar{u}=\pi / 2$ & $\mathrm{~F}$ & $\sin \bar{u}$ & $\operatorname{sign}\left(\sin u_{\text {ip } j}\right)$ & & \\
\hline $\bar{u} \in[\pi / 2, \pi)$ & $\mathrm{E}$ & $|\cos \bar{u}|$ & $\operatorname{sign}\left(\cos u_{\mathrm{ip} j}\right)$ & $g=-1$ & $g=1$ \\
\hline
\end{tabular}

Table 1 Feasible solution families depending on the aimed end-conditions

Table 2 All possible delta-v values for the triple-tangential fixed $\bar{u}$ solution scheme, once derived Signs and $g$ from Table 1

\begin{tabular}{lccc}
\hline Signs & $\delta \tilde{v}$ & $D$ & Remaining delta-v expressions \\
\hline+-- & $\delta v_{1}$ & $12 b(p-1)$ & $\delta v_{2}=-(4 b L+3 g(q-1) B+3 b(q+1) A) / D$ \\
-++ & & & $\delta v_{3}=+(4 b L+3 g(q-\mathrm{p}) B+3 b(q+\mathrm{p}) A) / D$ \\
--+ & $\delta v_{3}$ & $12 b(q-p)$ & $\delta v_{1}=-(4 b L-3 g(p-1) B+3 b(p+1) A) / D$ \\
++- & & & $\delta v_{2}=+(4 b L-3 g(q-1) B+3 b(q+1) A) / D$ \\
-+- & $\delta v_{2}$ & $12 b(q-1)$ & $\delta v_{1}=-(4 b L+3 g(p-1) B+3 b(p+1) A) / D$ \\
+-+ & & & $\delta v_{3}=+(4 b L-3 g(q-p) B+3 b(q+p) A) / D$ \\
\hline
\end{tabular}

\section{Safety concept}

This section focuses on the safety (i.e., collision avoidance) of the relative orbit prescribed by the manoeuvre planner. Since the presented algorithm is meant for onboard autonomous applications, the passive safety concept, achieved by keeping a certain minimum intersatellite separation in the radial-cross-track plane, is adopted. Regarding the ROE framework, for almost no drifting relative orbits, passive safety is related to the $\delta e$ and $\delta i$ magnitudes and to the angular phasing $\phi=\varphi-\theta$ of the relative eccentricity and inclination vectors (D’Amico and Montenbruck, 2006). Whereas, a not null relative semi-major axis maps into a shift in the radial local direction.

By recalling the geometrical representation of the ROEs in the $\delta \mathbf{e}$ and $\delta \mathbf{i}$ planes, let us focus on the behaviour of the relative eccentricity and inclination vectors determined by the functioning of the local control and planning units. 
At the local level, the single out-of-plane manoeuvre moves the relative inclination vector directly from its starting (i.e., $\left.\delta \mathbf{i}_{0, i}\right)$ to the final place (i.e., $\delta \mathbf{i}_{F, i}$ ) prescribed for the $i^{\text {th }}$ reconfiguration. This is depicted in the left view of Figure 2. In the meantime, the relative eccentricity vector covers the aimed jump in three steps, displaced along the direction of $\Delta \delta \mathbf{e}_{i}$ vector (see the right part of Figure 2). If an optimal solution is available, all the locations are contained in the $\Delta \delta \mathbf{e}_{i}=\delta \mathbf{e}_{F, i}-\delta \mathbf{e}_{0, i}$ segment. On the contrary, if the change in the shape of the orbit is not the dominant effect of the $i^{\text {th }}$ reconfiguration or if the control window is not lasting enough to support an optimal scheme, at least an intermediate relative eccentricity vector location resides outside the segment $\Delta \delta \mathbf{e}_{i}$. However, by selecting the minimum delta-v option the length of the realised total relative eccentricity vector variation is the minimum possible obtainable, thus points would be not spread too far from each other (Gaias and D'Amico, 2014).

At the planning level, equation (3) entails selecting intermediate $\mathbf{P}_{i}$ so that the total length to reach $\mathbf{P}_{\mathrm{F}}$ is minimised. Thus, the obtained configurations lay as most as possible aligned to the $\mathbf{P}_{\mathrm{F}}-\mathbf{P}_{0}$ line. Therefore, their projections on the unit-circle in the $\delta \mathbf{e} / \delta \mathbf{i}$ plane condense in a limited portion of the space.

Taking into account all these considerations, whenever a safe $\mathbf{P}_{\mathrm{F}}$ (i.e., $\phi_{\mathrm{F}} \approx k \pi$ and $k \in \mathbb{Z}$ ) is reached from a $\mathbf{P}_{0}$ with similar characteristics (i.e., $\phi_{0} \approx \phi_{\mathrm{F}}$ ) than the reconfiguration is passively safe during the whole duration of the reconfiguration, provided that the drifts are small enough (i.e., the reconfiguration takes place over a long enough time horizon). On the other hand, in the specific scenario of a far-range rendezvous to a non-cooperative target starting from an unsafe $\phi_{0}$, a double stage strategy can be exploited being $\mathbf{P}_{\mathrm{F}}$ a design parameter of the reconfiguration. First, $\phi_{0}$ can be corrected when the along-track separation is considerable, by commanding a safe temporary final configuration. Subsequently, the approach can safely start towards an aimed $\mathbf{P}_{\mathrm{F}}$ closer to the target.

Figure 2 Geometrical representation of variations of relative inclination and eccentricity vectors, respectively in the $\delta \mathbf{i}$ and $\delta \mathbf{e}$ planes
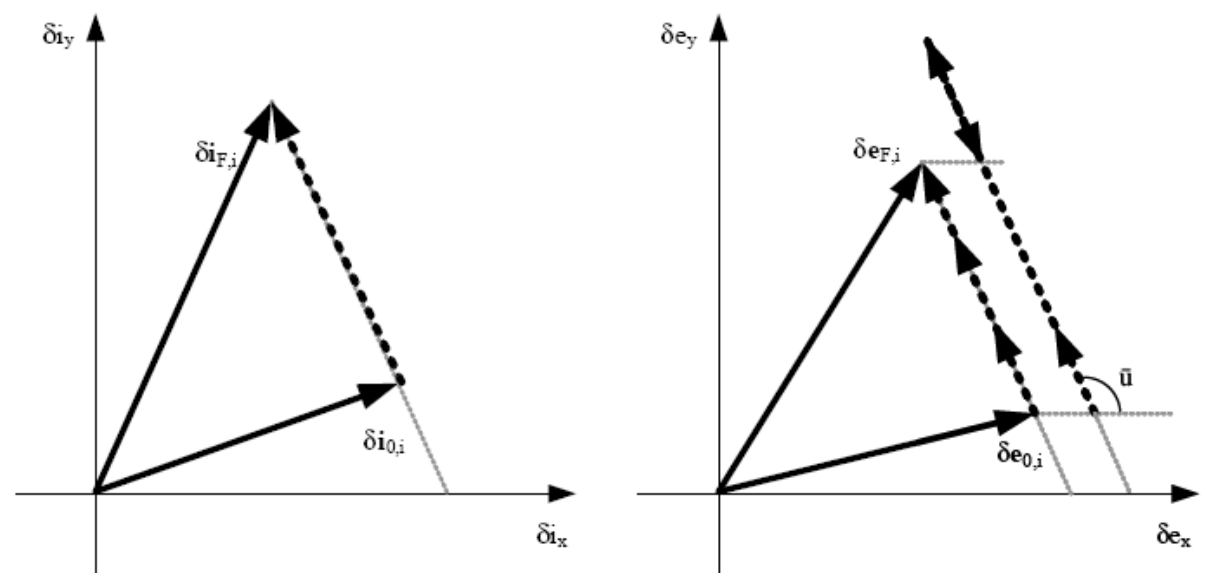


\section{Overview of the planner's implementation}

This section summarises how the algorithms described so far are implemented into the manoeuvre planner, and provides a broad introduction of the remaining tasks appearing in the architecture of Figure 1.

\subsection{Scheduling: Time Constraints Handling}

According to the planner interfaces shown in Figure 1, among the initial inputs, the user provides a list of times limiting periods in which it is not possible to schedule manoeuvres due to possible requirements of the space segment (see Section 2). Moreover, when in maximum observability mode, the user can define time barriers before which some manoeuvres shall occur (see Section 2.1). These pieces of information are handled by a scheduling functionality to determine the initial and final times of each interval in which it is allowed performing manoeuvres.

The basic reason of accomplishing the scheduling task at first is related to the fact that this planner is meant for autonomous onboard use. Therefore, from a computational point of view, it is simpler and more deterministic to directly search for a solution of the constrained problem instead of verifying and restoring the feasibility a posteriori. In the meantime, the complexity of the constrained problem is mitigated by the fact that, in realistic operational scenarios, the occurrence of manoeuvre exclusion windows affects a minimal part of the available time frame. Moreover, the planner under discussion exploits analytically derived manoeuvring schemes.

Concerning the implementation logic, in the case that any free time interval among two forbidden regions is shorter than two orbital periods, such portion of the schedule is merged to the bordering forbidden part. That is motivated from how the local control function operates: the fastest in-plane reconfiguration exploits a train of three half-orbital-period spaced impulses; the out-of-plane manoeuvre has to be distant enough from each of them to fulfil the manoeuvres' spacing time constraints. Finally, a forbidden time region is also automatically placed after each re-plan time $t_{0}$ to prevent scheduling early manoeuvres that would violate the time to first manoeuvre constraint.

\subsection{Plan generation}

By recalling Section 2.1, when in minimum delta-v mode the planning problem can be skip, since starting and aimed ROE sets are directly forwarded to the local control module. If in maximum observability mode, instead, the list of end-times of the control windows outputted by the scheduler is used by the planning unit. It computes the correspondent intermediate configurations $P_{i}$ that have to be established in order to achieve the final ROE state at the final reconfiguration time in a delta-v minimum way, according to the algorithm described in Section 3.

\subsection{Local control and manoeuvres' placement}

The effective placement of the manoeuvres within the available time frame embodies the concluding action of the planner. This is repeated for each intermediate reconfiguration, since it finalises the local control problem activity. Regarding the implementation, at first 
the in-plane manoeuvres' sequence is allocated. Consequently, the remaining out-ofplane burn is placed in accordance with the manoeuvres' spacing time constraints.

The placement of the in-plane manoeuvres is performed by selecting one manoeuvre scheme among the multiple available possibilities. As explained in Section 4, in fact, when an in-plane reconfiguration is performed through three tangential impulses multiple solutions are feasible, depending on the effective spacing between the burns (see Tables 1 and 2). The logic to perform such choice is presented in Figure 3. According to it, all schemes of the limited set of feasible solutions are sequentially processed: it is selected the option that successfully terminates that logical tree. In minimum delta-v mode a feasibility check (gray dashed box) has to be performed prior to the remaining evaluations. Only solutions whose manoeuvres are located in constraints-free regions can proceed. Further, minimum delta-v options are preferred. In case of multiple equal delta-v options it is preferred the solution that is characterised by a wider spacing between two of the three burns. This is motivated by the preference to leave more freedom to the remaining actions of the planner. Thus, by-product of the process is the end-time of the time interval dedicated to the out-of-plane burn $t_{i, \mathrm{oop}}$. It is emphasised that, a solution is always found due to the fact that the scheduler does not allow having manoeuvring windows shorter than two orbital periods.

Figure 3 Logic of the in-plane manoeuvres' scheme selection (see online version for colours)

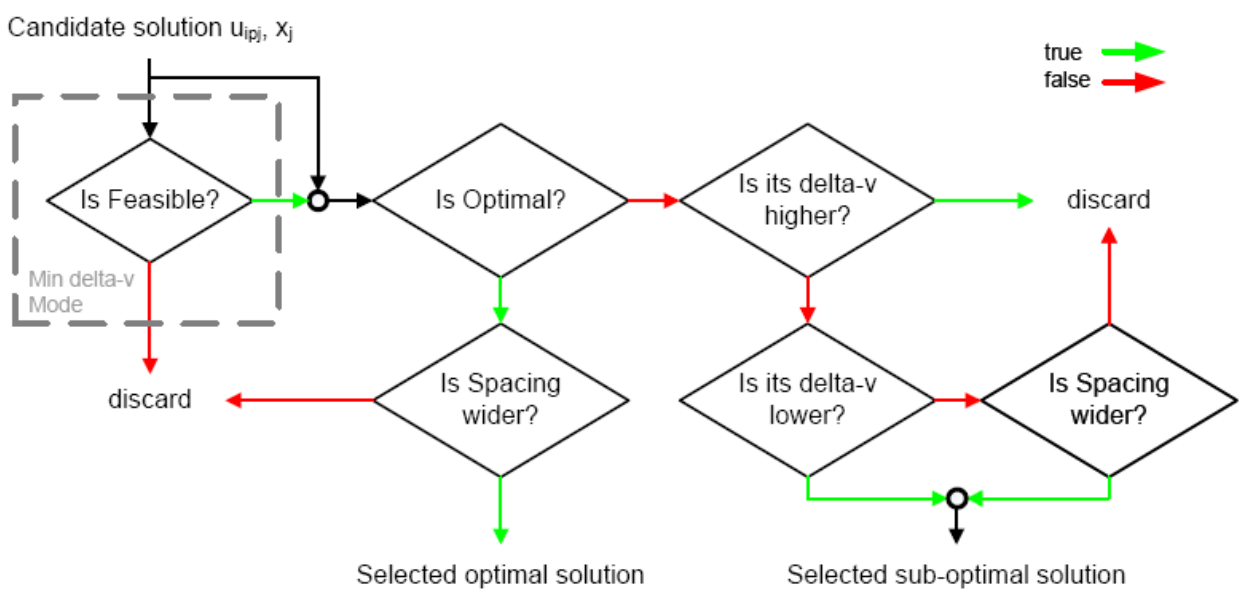

In conclusion, for each intermediate reconfiguration the out-of-plane manoeuvre is scheduled during the orbital period time that ends at $t_{i, \mathrm{oop}}$, according to equation (20). Nevertheless, when the whole intermediate reconfiguration horizon is particularly short, it can happen that the required $u_{\text {oop }}$ lays very close to $\bar{u}$, thus violating the manoeuvres' spacing time constraint. In this case, the planner merges the out-of-plane burn to a close in-plane manoeuvre and, at that argument of latitude, equation (20) is not respected anymore. The inconsistency is corrected by applying a second minor out-of-plane correction to another in-plane manoeuvre of the scheme to satisfy:

$$
\left\{\begin{array}{l}
n a \Delta \delta \tilde{i}_{x}=\delta v_{\mathrm{oop}, 1} \cos \left(u_{\mathrm{ip}, \mathrm{i}}\right)+\delta v_{\mathrm{oop}, 2} \cos \left(u_{\mathrm{ip}, \mathrm{j}}\right) \\
n a \Delta \delta \tilde{i}_{y}=\delta v_{\mathrm{oop}, 1} \sin \left(u_{\mathrm{ip}, \mathrm{i}}\right)+\delta v_{\mathrm{oop}, 2} \sin \left(u_{\mathrm{ip}, \mathrm{j}}\right)
\end{array}\right.
$$


being $i$ and $j$ the indexes of two selected in-plane manoeuvres of the scheme. Therefore, the resulting control sequence consists of three burns: two of them present components both in tangential and normal directions.

\section{Example of a rendezvous}

This section is intended to show the functioning of the manoeuvre planner. To this end, a representative test case of a rendezvous is defined and discussed.

The considered scenario involves a servicer satellite that has to approach a target by moving from an initial separation of circa $10 \mathrm{~km}$ to a final one of $3 \mathrm{~km}$. The details of the initial and final conditions in ROEs are presented in Table 3: $\mathbf{P}_{\mathrm{F}}$ is a bounded relative orbit with anti/parallel $\delta \mathbf{e}$ and $\delta \mathbf{i}$. The initial relative orbit $\mathbf{P}_{0}$ is characterised by a bigger size ( $\delta e$ and $\delta i$ magnitudes) than the aimed final one. Moreover, it presents a not exact anti/parallel configuration due to not null $x$ components of both relative eccentricity and inclination vectors.

The generation of the plan occurs at a time $t_{0}$ in which the mean argument of latitude of the servicer is 0 degrees. At that time, the servicer satellite is on a circular orbit $500 \mathrm{~km}$ high with 98 degrees of inclination. The approach has to be covered in 18 orbital periods that is approximately in 28 hours. At the height of the servicer spacecraft, the atmospheric density is equal to $1 \mathrm{~g} / \mathrm{km}^{3}$ for mean solar flux conditions [Harris Priester model (Montenbruck and Gill, 2001)] and the relative velocity is circa $7.6 \mathrm{~km} / \mathrm{s}$. In the example treated, it is assumed that the target has a ballistic coefficient $B$ of $0.01 \mathrm{~m}^{2} / \mathrm{kg}$ and the differential ballistic coefficient $\Delta B / B$ is $2 \%$.

Let us consider that for any reason the user commands to the spacecraft to avoid manoeuvring respectively from 5 to 7 and from 12 to 14 orbits after the plan generation time that occurs at orbit 1 . Moreover, in view of the eventual utilisation of the maximum observability mode, he commands that a reconfiguration shall occur within the first four orbital periods starting from $t_{0}$. The scheduling function merges this last requirement with the first manoeuvre exclusion window, since the time comprised between these two events is shorter than two orbital periods. As a result, the rendezvous horizon presents two time slots in which no manoeuvres can be scheduled, corresponding to the regions shadowed in gray in Figure 4.

In the example treated both time to first manoeuvre and time spacing between manoeuvres are set equal to 10 minutes.

When the planner is set to minimum delta-v mode, the manoeuvres' profile reported in the left view of Figure 4 is generated. In particular, the reconfiguration happens in one step over the complete time horizon thus requiring four manoeuvres. Since the most relevant orbit change is in $\|\Delta \delta \mathbf{e}\|$, the optimality criterion based on the lower bound of the delta- $\mathrm{v}$ is applicable. The schemes of Tables 1 and 2 generate 216 feasible optima solutions that have to be pruned through the logic of Figure 3. It is emphasised that this approach constitutes a generalisation of the solution proposed in Eckstein (1992) to cope with the presence of time constraints. Instead of moving the mean argument of latitude of a manoeuvre scheduled in a forbidden time region, though achieving a sub-optimal result, here alternative optimal and feasible manoeuvring schemes are systematically searched. In conclusion, the total commanded delta-v amounts to circa $0.20 \mathrm{~m} / \mathrm{s}$, and corresponds to the absolute minimum cost for this rendezvous scenario. 
The right view of Figure 4 shows the manoeuvres' plan generated by the planner when operating in the maximum observability mode. There the intermediate configurations $\mathbf{P}_{1}$ and $\mathbf{P}_{2}$ of Table 3 are to be achieved respectively within 4 and 12 orbital periods after $t_{0}$. According to the required variations of ROEs, for each of these three reconfigurations $\|\Delta \delta \mathbf{e}\|$ is not the dominant change to be achieved. Hence, the local control can accomplish only suboptimal solutions. Among the multiple available solutions, the best ones (more convenient in terms of delta-v) have been selected according to the logic depicted in Figure 3. As a result, tangential (green) and normal (blue) manoeuvres are automatically scheduled, in agreement with manoeuvring spacing constraints. The total realised delta-v amounts to circa $0.217 \mathrm{~m} / \mathrm{s}$; as expected it is slightly higher than in the previous case.

Table 3 Example's scenario and intermediate configurations

\begin{tabular}{lccccccc}
\hline Input & Intermediate & \multicolumn{7}{c}{$a \delta \alpha[\mathrm{m}]$} \\
\hline $\mathbf{P}_{0}$ & & 5 & 10,000 & -50 & -250 & -30 & 200 \\
& $\mathbf{P}_{1}$ & 54.6 & $9,814.2$ & -34.1 & -199.3 & -22.1 & 166.7 \\
& $\mathbf{P}_{2}$ & 48.1 & $5,714.2$ & -19.0 & -149.0 & -11.9 & 132.9 \\
& & 0 & 3,000 & 0 & -100 & 0 & 100 \\
\hline
\end{tabular}

Both the top views of Figure 4 focus on the $\delta \mathbf{e}$ and $\delta \mathbf{i}$ vectors configurations during the rendezvous. In particular, the relative eccentricity (marked with squares) and the relative inclination (marked with circles) unit vectors achieved after each manoeuvre are shown. The later the manoeuvre the lighter the colour of the marker. In agreement with the employed algorithm, the safe anti/parallel configuration is kept during the approach. Moreover the values of $\delta e$ and $\delta i$ together with the drift magnitudes $a \delta a$, shown in Figure 5 for the maximum observability solution, allow the relative trajectory being passive safe throughout the approach.

Figure 4 manoeuvres' plan in both the operative modes for the rendezvous of Table 3 (see online version for colours)

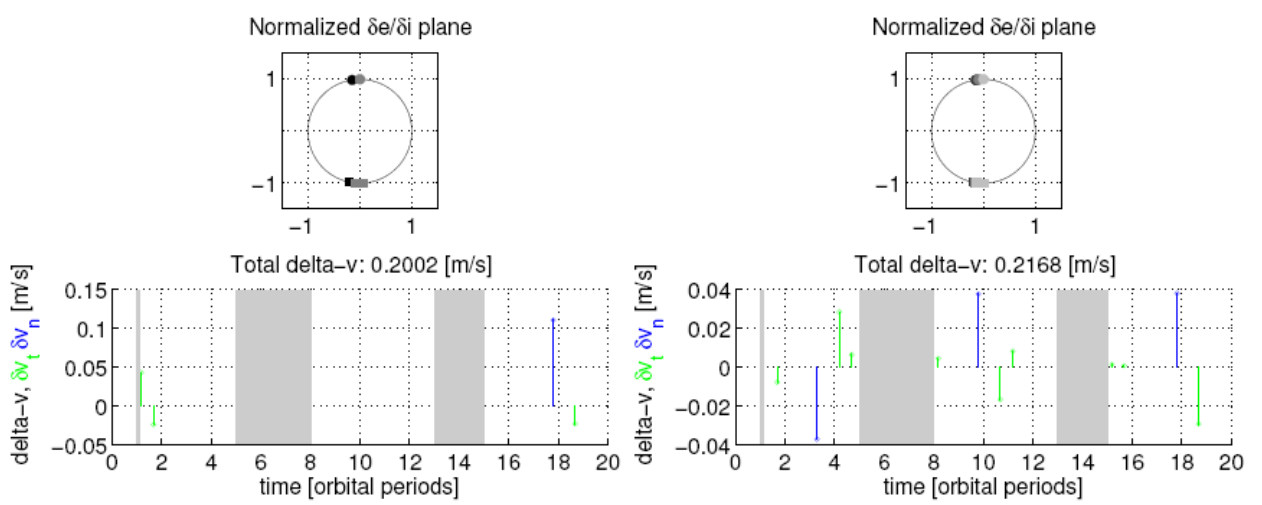

Finally, Figure 5 focuses on the behaviour over time of the ROEs during the rendezvous obtained in the maximum observability mode. Since we are focusing on a qualitative analysis and not on an accurate estimate of the control performance, the ROEs are simply propagated making use of the analytical model discussed in Section 3. Manoeuvres are 
introduced as instantaneous ROE discontinuities at the proper mean arguments of latitude.

The left view of Figure 5 reports the intermediate $\delta \mathbf{e}$ and $\delta \mathbf{i}$ locations which, as expected, distribute around the directions of their respective total aimed variations. The right view, instead, shows how the inter-satellite separation reduction is obtained by establishing a certain relative semi-major axis at the beginning of the plan. Such drift is cancelled in proximity of the time at which the aimed final mean along-track separation $a \delta \lambda_{F}$ is reached. This behaviour is determined by the attempt to employ the minimum possible delta-v; hence, exploiting at maximum the available time horizon. In realistic applications this aspect shall be mitigated with a proper strategy of setting $\mathbf{P}_{\mathrm{F}}$, as the chain of navigation and control errors can lead to an overshooting in the along-track direction.

The rendezvous just discussed provides an example of the manoeuvres' plan generated by the planner, depending on the operative mode. In order to assess the overall control accuracy, instead, the complete chain of relative navigation filter, manoeuvre planner, and thrusters' system has to be considered. Regarding the implementation envisioned for AVANTI, the manoeuvres' plan is updated as soon as the first sub-reconfiguration is achieved (i.e., at latest after having performed the first four manoeuvres of a plan). In this way, in fact, the remaining portion of the manoeuvres' plan is refined taking into account a more recent estimation of the relative state and the effect of the execution errors of the manoeuvres already performed.

Figure 5 Rendezvous propagation in the ROE space (see online version for colours)
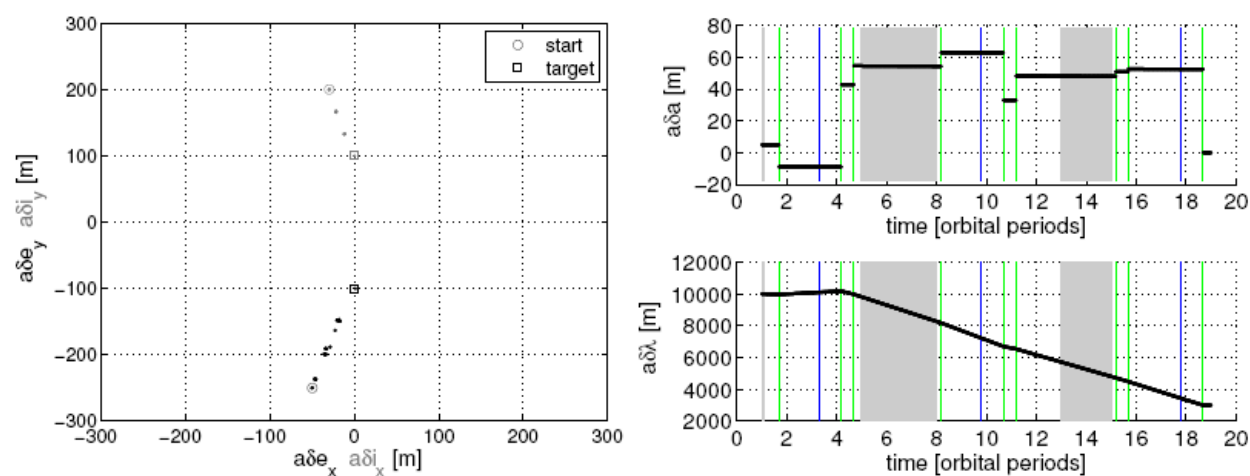

\section{Conclusions}

This work addresses the design of a manoeuvres' planner for large reconfigurations of satellites formation flying in near-circular orbit. The presented algorithms constitute the core part of the manoeuvre planner module developed for the AVANTI experiment, to be conducted in the frame of the DLR FireBird mission.

Simplicity and determinism constituted driver features in the algorithm development. This turned out into the exploitation of the geometrical meaning of the ROEs and of the existence of analytical solutions for accomplishing stepwise reconfigurations in a delta-v minimum manner. Despite this, the planner is able to meet important operational 
requirements like passive safety during a rendezvous. Moreover, it offers the capability to introduce time constraints that prevent executing manoeuvres in certain portions of the schedule.

\section{Acknowledgements}

This work was done while the co-author, Simone D'Amico, was affiliated with the German Aerospace Center (DLR), GSOC/Space Flight Technology Department.

\section{References}

Ardaens, J-S., D'Amico, S. and Fischer, D. (2011) 'Early flight results from the TanDEM-X autonomous formation flying system', 4th International Conference on Spacecraft Formation Flying Missions \& Technologies (SFFMT), St-Hubert, Quebec, Canada, 18-20 May.

Bodin, P., Noteborn, R., Larsson, R., Karlsson, Th., D’Amico, S., Ardaens, J-S., Delpech, M. and Berges, J-C. (2012) 'PRISMA formation flying demonstrator: overview and conclusions from the nominal mission', 35th Annual AAS Guidance and Control Conference, AAS 12-072, Breckenridge, Colorado, 3-8 February.

Clohessy, W.H. and Wiltshire, R.S. (1960) 'Terminal guidance system for satellite rendezvous', Journal of the Aerospace Sciences, Vol. 27, No. 9, pp.653-658.

D'Amico, S. (2010) Autonomous Formation Flying in Low Earth Orbit, PhD thesis, Technical University of Delft.

D'Amico, S. and Montenbruck, O. (2006) 'Proximity operations of formation flying spacecraft using an eccentricity/inclination vector separation', Journal of Guidance, Control, and Dynamics, Vol. 29, No. 3, 2006, pp.554-563.

D'Amico, S., Ardaens, J-S. and Larsson, R. (2012) 'Spaceborne autonomous formation-flying experiment on the PRISMA mission', Journal of Guidance, Control, and Dynamics, Vol. 35, No. 3, pp.834-850.

D’Amico, S., Ardaens, J-S., Gaias, G., Benninghoff, H., Schlepp, B. and Jørgensen, J.L. (2013) 'Noncooperative rendezvous using angles-only optical navigation: system design and flight results', Journal of Guidance, Control, and Dynamics, Vol. 36, No. 6, pp.1576-1595.

Eckstein, M.C. (1992) Generalized Maneuver Planning for Station Keeping, Station Acquisition, Longitude Transfer and Reorbiting of Geostationary Satellites, Technical Note, GSOC TN 926 , Deutsches Zentrum für Luft- und Raumfahrt.

Gaias, G. and D'Amico, S. (2014) 'Impulsive maneuvers for formation reconfiguration using relative orbital elements', Journal of Guidance, Control, and Dynamics [online] doi: http://arc.aiaa.org/doi/abs/10.2514/1.G000189 (accessed 25 April 2014).

Gaias, G., Ardaens, J-S. and D'Amico, S. (2014a) 'The autonomous vision approach navigation and target identification (AVANTI) experiment: objectives and design', 9th International ESA Conference on Guidance, Navigation \& Control Systems, Porto, Portugal, 2-6 June.

Gaias, G., D'Amico, S. and Ardaens, J-S. (2014b) 'Angles-only navigation to a noncooperative satellite using relative orbital elements', Journal of Guidance, Control, and Dynamics, Vol. 37, No. 2, pp.439-451, DOI: 10.2514/1.61494.

Ichimura, Y. and Ichikawa, A. (2008) 'Optimal impulsive relative orbit transfer along a circular orbit', Journal of Guidance, Control and Dynamics, Vol. 31, No. 4, pp.1014-1027.

Larsson, R., Berge, S., Bodin, P. and Jönsson, U. (2006) 'Fuel efficient relative orbit control strategies for formation flying rendezvous within PRISMA', Advances in the Astronautical Sciences, also 29th Rocky Mountain Guidance and Control Conference, AAS Paper 2006-025, Vol. 125, pp.25-40. 
Montenbruck, O. and Gill, E. (2001) Satellite Orbits-Models, Methods, and Applications, Springer-Verlag, Heidelberg, Germany.

Reile, H., Lorenz, E. and Terzibaschian, T. (2013) 'The FireBird mission - a scientific mission for Earth observation and hot spot detection', Small Satellites for Earth Observation, Digest of the 9th International Symposium of the International Academy of Astronautics, Wissenschaft und Technik Verlag, Berlin, Germany.

Schaub, H. and Alfriend, K.T. (2001) 'Impulsive feedback control to establish specific mean orbit elements of spacecraft formations', Journal of Guidance, Control and Dynamics, Vol. 24, No. 4, pp.739-745, DOI: 10.2514/2.4774.

Tillerson, M. and How, J. (2002) 'Advanced guidance algorithms for spacecraft formation-keeping', Proceedings of the American Control Conference, Vol. 4, pp.2830-2835, DOI: 10.1109/ACC.2002.1025218.

Tillerson, M., Inalhan, G. and How, J. (2002) 'Co-ordination and control of distributed spacecraft systems using convex optimization techniques', Int. J. Robust Nonlinear Control, Vol. 12, Nos. 2-3, pp.207-242, DOI: 10.1002/rnc.683.

Vaddi, S.S., Alfriend, K.T., Vadali, S.R. and Sengupta, P. (2005) 'Formation establishment and reconfiguration using impulsive control', Journal of Guidance, Control and Dynamics, Vol. 28, No. 2, pp.262-268, DOI: 10.2514/1.6687.

Yamanaka, K. and Ankersen, F. (2002) 'New state transition matrix for relative motion on an arbitrary elliptical orbit', Journal of Guidance, Control and Dynamics, Vol. 25, No. 1, pp.60-66, DOI: $10.2514 / 2.4875$. 